\title{
Routing Optimization Based on OSPF in Multi-Layer Satellite Network
}

\author{
Mingjiang $\mathrm{FU}^{1}$, Bingli GUO, Hai YANG, Chengguang PANG and Shanguo HUANG \\ State Key Laboratory of Information Photonics and Optical Communications, \\ Beijing University of Posts and Telecommunications, Beijing, 100876, China
}

\begin{abstract}
Multi-layer satellite network has become a hot spot for its wider coverage and higher bandwidth level. However, due to the frequent link changes and complexity of network, it is hard to find out a mechanism to handle well on long delay and high packet loss level. This paper proposes an optimized OSPF protocol called OOWLP to eliminate unnecessary routing convergence to optimize the packet loss level and delay ultimately. Link plan table, which records link contacting plan, will be used to update the link state database periodically so that we can eliminate the flooding procedure caused by scheduled link changes. On the other hand, Constrained Shortest Path First (CSPF) will be used to get business differentiated routes in multi-layer satellite network to optimized the throughput capacity in congestion scenario. We divide the sending packets into different businesses and get the routes for each business with longer duration limited by remaining bandwidth. Simulation results show that in normal scenario, average packet loss rate and delay performance are improved $17.42 \%, 51.44 \mathrm{~ms}$ respectively, average packet loss rate and throughput capacity performance are optimized $79.05 \%, 9.81 \mathrm{Mbps}$ respectively in congestion scenario compared to standard OSPF. As a result, the proposed mechanism is able to shorten the average delay and lower the packet loss level in multi-layer satellite network.
\end{abstract}

Keywords. Optimized OSPF with Link Plan (OOWLP), Multi-layer Satellite Network (MLSN), Link Plan Table (LPT), Constrained Shortest Path First (CSPF)

\section{Introduction}

As an essential member in the $6 \mathrm{G}$ version, satellite network will be integrated with the terrestrial networks to provide users ubiquitous access capability [1]. With the development of satellite communication technology, single-layer satellite networks cannot meet the gradually increased needs of coverage area, Quality of Services (QoS) and robustness. Meanwhile multi-layer satellite networks (MLSN) strike into people's sight [2-4], which can provide a more efficient network with better performance than single-layer networks [5]. Link QoS will affect the data interchange in the inter-satellite link deeply. To meet the requirements, the satellite network needs effective QoS guarantee [6].

A distributed contact plan design scheme is proposed to divide the complete contact information into several partial parts to reduce its complexity [7], but frequent link changes still lead to severe packet loss. Korçak [8] proposed a multi-state virtual network

\footnotetext{
${ }^{1}$ Corresponding Author, Beijing University of Posts and Telecommunications, Beijing, 100876, China; E-mail: 2019111636@bupt.edu.cn
} 
topology to give us the new efficient handover mechanisms to reduce packet loss, but this scheme cannot handle packet loss well in link congestion scenario. Heuristic algorithms [9-11] lead us to optimize variety of QoS parameters, but it is difficult to apply to MLSN practically for the complexity of the mechanism and long duration of convergence. All in all, these mechanisms have their advantages in normal scenario, but never mentioned in congestion scenario. Enlightened from these mechanisms, we need a mechanism with fine QoS in both normal and congestion scenarios.

In this paper, Optimized OSPF with Link Plan (OOWLP) is proposed to reduce packet loss caused by frequent link change in satellite application scenarios. Link Plan Table (LPT) is introduced to store scheduled link information to update link state periodically. Furthermore, the data business is allocated to different routes according to the remaining link bandwidth and effective link duration to get the optimal transmission path in link congestion scenario, which will be mentioned in Constrained Shortest Path First (CSPF) algorithm herein below. Simulation results show that the enhanced routing strategy can eliminate unnecessary routing convergence to lower the packet loss level and select the optimal transmission path for MLSN routing selection process to shorten the average delay.

The remainder of this paper is organized as follows. Section 2 introduces the construction of multi-layer satellite constellation, including the generation of link plan table. Main strategies involving OOWLP and CSPF are amply presented in Section 3. Simulation results are shown in Section 4. In Section 5, conclusions are provided.

\section{Multi-layer Satellite Constellation Construction}

Generally, the multi-layer satellite network (MLSN) may consist of Inclined GeoSynchronous Orbit (IGSO) satellites, Medium Earth Orbit (MEO) satellites and Low Earth Orbit (LEO) satellites [12]. The contacting plan design procedure is also an essential approach for MLSN operation. AGI System Tool Kit (STK) [13] is a powerful tool to obtain the visibility data for a given MLSN constellation. With the visibility information, a time-varying topology could be generated through various algorithms, which records link adjacency relationship associated with their start and end time. Finally, link state information is extracted from the time-varying topology structure and composes the LPT, which contains the nodes' link connection state and effective time period to give us the basis of link update later. Our simulations will be set up in Omnet++ simulation environment, which can be freely used for academic research with an open simulation core and supporting alterable network topology [14].

\section{Routing Optimization Strategy for MLSN}

\subsection{OOWLP}

In the original rule of OSPF, neighbors are detected by the hello message interaction process, stored in the Interface Neighbor Information Structure (INIS) and gathered to constitute a Link State Advertising (LSA) which is stored in the Link State Database (LSDB). If we apply the standard OSPF directly to our MLSN, frequent link changes will cause the route convergence frequently, leading to long period of network instability, packet loss and longer hops. 
To deal with the above-mentioned defects, we propose the OOWLP. As mentioned before, LPT is designed to preserve the link plan information of the whole network for a period of time. The ground station will send the new LPT periodically to the satellites who directly connect to it. Meanwhile the satellites will flood the compressed LPT to other satellites in MLSN.

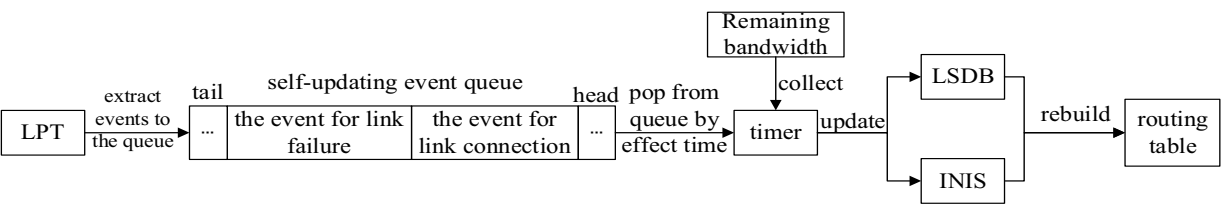

LPT: Link Plan Table LSDB: Link State Database INIS: Interface Neighbor Information Structure LSA: Link State Advertising

Fig 1. OOWLP updating process.

When any satellite gets the new LPT, the update procedures will come into operation as shown in Fig 1. Firstly, the events are extracted from LPT to update the LSDB and INIS regularly. These events are split into two types based on the LPT entry, including the event for link connection which relied on effective time start, and the event for link disconnected which is based on effective time end. The flag bit called "conn or intrp" is used to distinguish between the two types. Furthermore, a timer is set to handle the events who reach the effective time. For the link connection event, if the event matches an INIS, the neighbor IP and neighbor interface IP in the INIS should be replaced with the corresponding two parts in the event. In addition, the LSA, who matches the event, should update the link id from the neighbor IP of the event. As for the link failure event, it has the same processes to the previous, but we will clear the neighbor information in the corresponding INIS and LSA. Also, we will collect the link remaining bandwidth and add to the corresponding part in LSDB. Finally, we will rebuild the routing table.

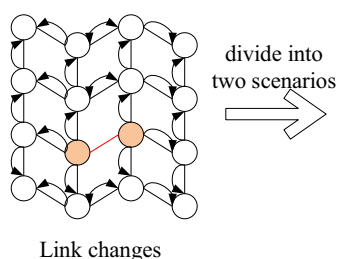

Link changes

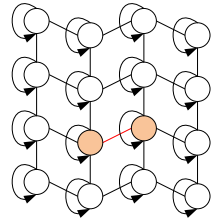

Scheduled link changes
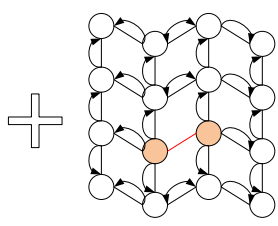

Emergency link changes
The node who

detected link changes

- Scheduled link changes

$\bigcirc$ Flooding procedure

Self-updating procedure

Fig 2. Flood process modification.

Meanwhile, we revise the judgement of flooding procedure as shown in Fig 2. In the original mechanics, if the on-off action appeared in one link, the two nodes of the link will flood the updating message to other nodes in the network. It will cost much of time on route convergence and occupying parts of the link resources. As a result, we divide the link changes to two kinds of situation, for most of time the link change can be predicted, and rare of time the link change is unpredictable. As mentioned before, we periodically update the LSDB and INIS by the LPT. Thus, we restrain the predictable flooding, and keep the link monitoring active for the emergency situation. 


\section{2. $C S P F$}

Dijkstra algorithm is the core routing algorithm in the OSPF protocol. Based on the link information of the whole network in LSDB, the shortest path from the local node to other nodes in the network can be calculated by Dijkstra algorithm and added into the routing table. However, because of the resource constraints in space, link congestion problem will easily happen if the routes we choose are always in shortest hops. So, we put forward the CSPF on allocating data business to different routes which meet the business needs.

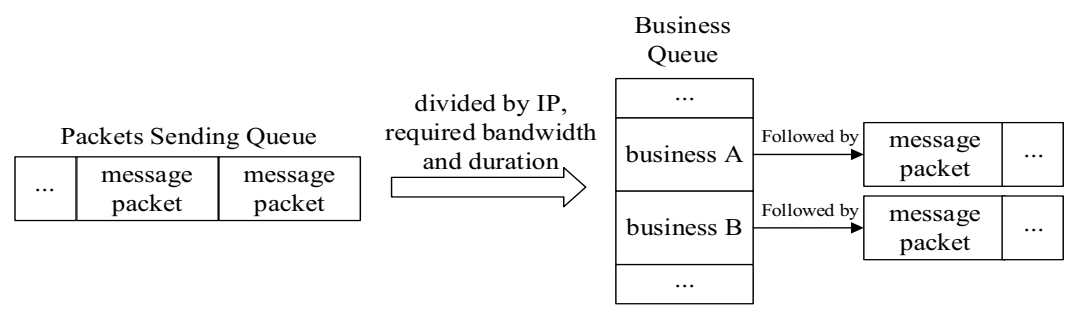

Fig 3. Divide packets from sending queue.

Then, we divide the packets from packet sending queue into different kinds of business, and put each packet into the corresponding business item of business queue. If the packet belongs to new business, record the IP information including source IP and destination IP, required bandwidth and duration of the packet to form a new business item into the business queue, as shown in Fig 3. Afterwards, we send the packet by business type.

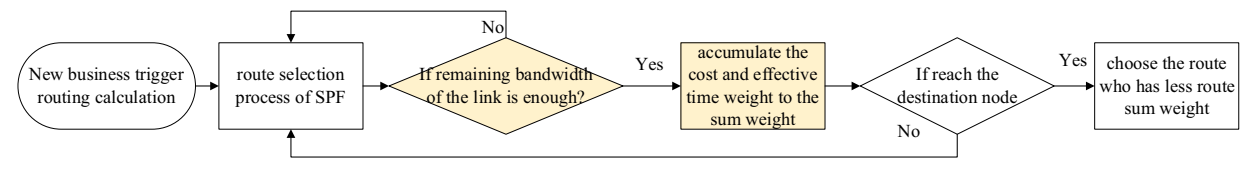

(a) CSPF route calculation procedure.

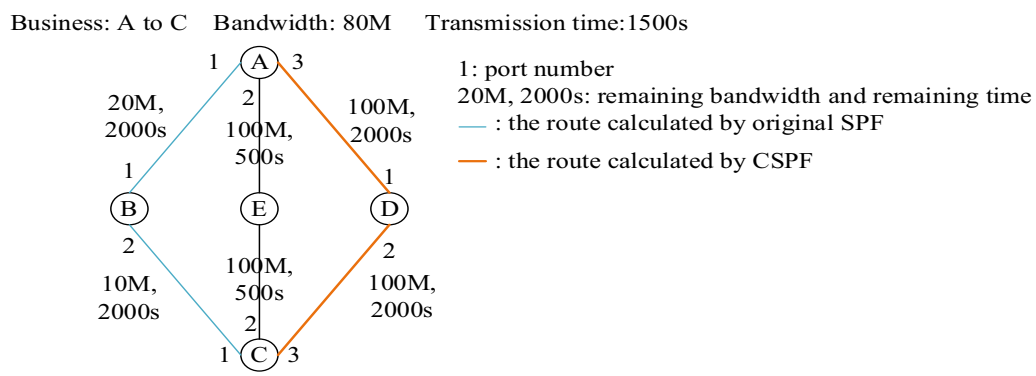

(b) CSPF route calculation compared with original SPF.

Fig 4. The principle of CSPF

Next, we set up the CSPF route calculation procedure based on the SPF algorithm as shown in Fig 4 (a). Route paths are calculated based on the remaining bandwidth and remaining effective time into picking up the links. The link we picked should meet the required bandwidth of the business, and the shortest remaining effective time of the route will be as long as possible. Therefore, we add the weight of effective time into the 
accumulation of the link cost. The remaining effective time is divided by an appropriate value as the weight of the effective time to keep balance with the origin cost, here we choose the maximum remaining effective time as the value to divide each remaining effective time of the link. In Fig 4 (b), we provide an example to compare our CSPF with the original SPF. Here we set a data business from node A to node $\mathrm{C}$, requiring bandwidth $80 \mathrm{M}$ and transmission time 1500s. Through the original SPF algorithm, we get the route path "A->B->C" with lack bandwidth, which may lead to packet loss. By CSPF, the route path will be replaced to "A->D->C", which has enough bandwidth and longer duration.

\section{Simulation and Results}

In this simulation, we build out our MLSN in Omnet++ simulator as Chapter 2 mentioned. And we apply the two sets of routing algorithm to our scenario, one is the standard OSPF and the other is CSPF. Our OOWLP is used to eliminate unnecessary routing convergence and CSPF is to get the business differentiated routes with enough bandwidth. To verify the superiority of the OOWLP, we use the broadcast and multicast business to our scenario and compare the standard OSPF to our OOWLP through average delay and packet loss rate in different contract awarding frequency as shown in Fig 5 (a). As for CSPF, we use the partial congestion business and compare the standard OSPF to our CSPF through throughput capacity and packet loss rate in different sending rate, as shown in Fig 5 (b).
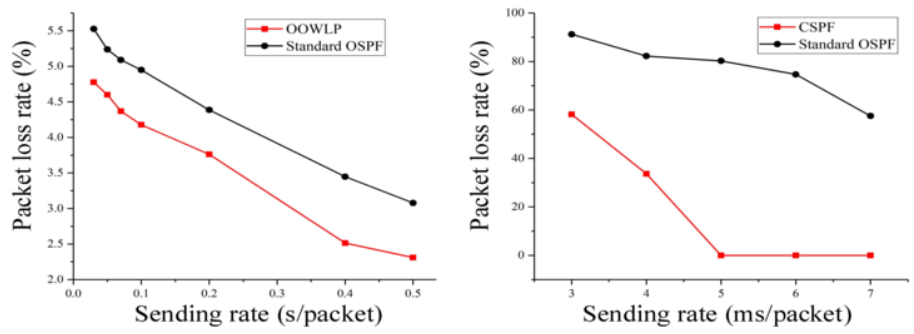

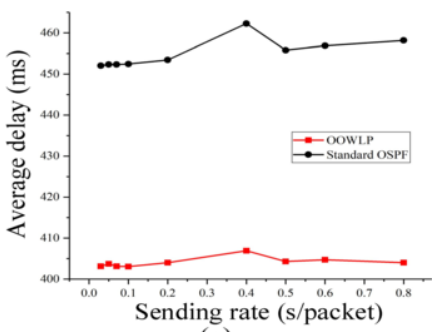

(a)

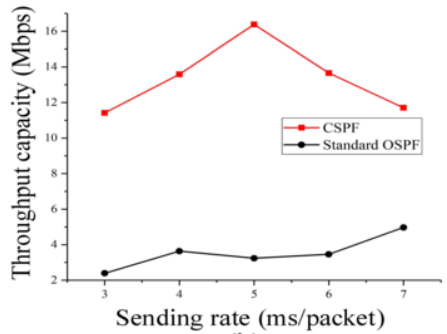

(b)

Fig 5 (a) OOWLP and Standard OSPF performance comparison in normal sending rate, (b) CSPF and Standard OSPF performance comparison in the congestion scenario

As shown in Fig 5 (a), the average packet loss rate in OOWLP is lower than the standard OSPF, which decrease $17.42 \%$, and average delay decrease $51.44 \mathrm{~ms}$. Our 
OOWLP eliminates unnecessary routing convergence to reduce the effect on packet loss and delay. In Fig 5 (b), the average packet loss rate of CSPF is improved with $79.05 \%$ and average throughput capacity is increased for $9.81 \mathrm{Mbps}$ compared to the standard OSPF. Because data businesses are divided to the business differentiated routes to reduce the probability of link congestion and increase the throughput.

\section{Conclusion}

In this paper, we propose OOWLP strategy to eliminate the unnecessary routing convergence and enhance the network performance. Besides the CSPF is raised to get the business differentiated routes to obtain the optimized transmission paths under the link congestion situation in MLSN. Simulation results show that the OOWLP can reduce the packet loss rate and delay effectively. Furthermore the CSPF can decrease the packet loss rate and improve the throughput capacity in link congestion scenario.

\section{Acknowledgements}

This work was funded in part by BUPT innovation and entrepreneurship support program (2021-YC-A057), State Key Laboratory of Advanced Optical Communication Systems Networks, China.

\section{Reference}

[1] NetWorld 2020's White Paper, "SatCom WG: The role of satellite in 5G", July 2014, https://www.networld2020.eu/white-papers/

[2] Yu Zhang, Bo Wang, Bingli Guo, "A Research on Integrated Space-Ground Information Network Simulation Platform Based on SDN", publicshed in Computer Networks 188 (2021) 107821.

[3] Xiangming Zhu, Chunxiao Jiang, LinLing Kuang, "Capacity Analysis of Multi-layer Satellite Networks", published in 2020 International Wireless Communications and Mobile Computing

[4] Zhizhong Yin, long zhang. "Qos-Aware Multicast Routing Protocol for Triple-layered LEO/HEO/GEO satellite IP networks". IEEE, October 2010.

[5] ChaoYuan, Xiaoxiang Wang, "A Multicast Routing Algorithm for GEO/LEO Satellite IP Networks", in 2013 IEEE 11th International Conference on Dependable, Autonomic and Secure Computing.

[6] Tengyue Mao, Bin zhou, Zhengquan Xu, "A Multi-QoS Optimization Routing for LEO/MEO Satellite IP Networks", published in JOURNAL OF MULTIMEDIA, VOL. 9, NO. 4, APRIL 2014.

[7] Wenfeng Shi, Deyun Gao, Huachun Zhou, "Distributed Contact Plan Design for Multi-layer Satelliteterrestrial Network", published in China Communications, volume 15, issue 1, Jan.2018.

[8] Korçak, Ö. and Alagöz, F., "Virtual topology dynamics and handover mechanisms in Earth-fixed LEO satellite systems." Computer Networks, vol. 53, pp. 1497-1511, 2009.

[9] Fei Long, Naixue Xiong, Athanasios V. Vasilakos, "A sustainable heuristic QoS routing algorithm for pervasive multi-layered satellite wireless networks", Wireless Networks 16, 1657-1673 (2010).

[10] Y. H. Zhou; F. C. Sun; B. Zhang, "A Hierarchical and Distributed QoS Routing Protocol for Two-layered Satellite Networks", published in: The Proceedings of the Multiconference on "Computational Engineering in Systems Applications".

[11] Fei Long, Fuchun Sun, Zhian Yang, "A Novel Routing Algorithm Based on MultiObjective Optimization for Satellite Networks", published in JOURNAL OF NETWORKS, VOL. 6, NO. 2, FEBRUARY 2011.

[12] Wei Zhou,Yi-fan Zhu,Yao-yu Li,Qun Li,Qin-Zhang Yu, "Research on hierarchical architecture and routing of satellite constellation with IGSO-GEO-MEO network", published in "International Journal of Satellite Communications and Networking" volume 38, issue 2.

[13] Junqing Qi, Zhuoming Li and Gongliang Liu, "Research on Coverage and Link of Multilayer Satellite Network based on STK”, 2015 10th International Conference on Communications and Networking in China (China Com).

[14] OMNeT++ home page: http://www.omnetpp.org/. 